\title{
Tumor-associated fibroblasts promote the proliferation and decrease the doxorubicin sensitivity of liposarcoma cells
}

\author{
KAMRAN HARATI, ADRIEN DAIGELER, TOBIAS HIRSCH, FRANK JACOBSEN, BJÖRN BEHR, \\ CHRISTOPH WALLNER, MARCUS LEHNHARDT and MUSTAFA BECERIKLI \\ Department of Plastic Surgery, Burn Center, Hand Center, Sarcoma Reference Center, \\ BG-University Hospital Bergmannsheil, D-44789 Bochum, Germany
}

Received January 19, 2016; Accepted April 5, 2016

DOI: $10.3892 /$ ijmm.2016.2556

\begin{abstract}
The reasons for the distinct chemoresistance of liposarcomas and their high risk of local recurrence still remain unclear. Depending on the histological subtype of liposarcoma, first-line therapy with the cytostatic agent, doxorubicin, only achieves response rates of approximately $36 \%$. Approximatley $70 \%$ of all local recurrences develop in spite of complete surgical resection of the primary tumor with microscopically negative margins. In this study, we aimed to assess the influence of tumor-associated fibroblasts (TAFs) obtained from surgically removed liposarcomas on the well-established human liposarcoma SW872 cell line. Intratumoral TAFs were isolated from intermediate- and high-grade liposarcoma samples. The human liposarcoma cell line, SW872, was co-cultured with the corresponding TAFs or with dermal fibroblasts as a control. The proliferation (by BrdU assay), cell viability (by MTT assay) and sensitivity to doxorubicin (using the iCELLigence system) of the co-cultured SW872 cells were examined. The SW872 cells exhibited a significant increase in proliferation and viability when co-cultured with the TAFs. As detected by real-time cell analysis, the SW872 cells co-cultured with the TAFs exhibited a diminished response towards doxorubicin. Notably, co-culture with TAFs obtained from high-grade liposarcoma samples resulted in higher proliferation and increased chemoresistance than co-culture with TAFs obtained from intermediate-grade liposarcoma samples. The findings of the present study thus indicate that TAFs from liposarcomas enhance the proliferation and decrease the chemosensitivity of SW872 liposarcoma cells significantly compared with normal fibroblasts from the dermis. TAFs from more malignant liposarcomas promoted tumor cell proliferation and chemoresistance more strikingly than TAFs from less malignant liposarcomas. These data provide evidence
\end{abstract}

Correspondence to: Dr Kamran Harati, Department of Plastic Surgery, Burn Center, Hand Center, Sarcoma Reference Center, BG-University Hospital Bergmannsheil, Buerkle-de-la-Camp-Platz 1, D-44789 Bochum, Germany

E-mail: kamran.harati@t-online.de

Key words: soft tissue sarcoma, liposarcoma, tumor-associated fibroblasts, tumor microenvironment, doxorubicin, chemosensitivity for the influence of the tumor microenvironment on liposarcoma and support for further investigations in patients with different liposarcoma subentities, assessing the influence of TAFs on tumor progression.

\section{Introduction}

Soft tissue sarcomas are a heterogeneous group of solid malignant tumors which represent approximately $1 \%$ of all malignancies (1). Liposarcomas are the most common soft tissue sarcomas in adults, comprising approximately $20 \%$ of all soft tissue sarcomas $(2,3)$. The peak incidence of liposarcomas is between 52 and 56 years of age, and men are slightly more often affected than women $(3,4)$. Liposarcomas can occur throughout the body; however, 50-60\% of all liposarcomas are localized in the deep, subfascial soft tissues of the thighs $(3,5)$.

In patients with primary diagnosed liposarcoma without concurrent distant metastasis, the therapy of choice involves limb-sparing surgical resection with clear margins, usually followed by radiation treatment to decrease local recurrence $(6,7)$. In this context, the achievement of negative surgical margins in primary liposarcoma has been determined to be an important factor for improving local disease control (8-12). However, approximately $62-70 \%$ of all local recurrences develop in spite of complete surgical resection of the primary tumor with microscopically negative margins $(5,13,14)$.

Disseminated liposarcomas pose another therapeutic challenge due to the limited effectivity of the currently available treatment options. Notably, approximately $30 \%$ of all patients with liposarcoma develop distant metastases during the course of the disease $(3,15-19)$. In cases of distant metastatic disease, the median survival time is only $<10$ months (19). Doxorubicin, which has been the most frequently used chemotherapeutic agent in the treatment of metatstatic soft tissue sarcomas, only achieves response rates of $36 \%$ in liposarcoma as a whole (20). Concerning the four different histological subsets, well-differentiated and dedifferentiated liposarcomas display even worse response rates of 12 and $13 \%$, respectively (21). Pleomorphic liposarcomas represent the most chemoresistant subentity, with response rates of only 5\% towards doxorubicin, while myxoid/round cell liposarcomas are considered the most chemosensitive subgroup, displaying response rates of $44-48 \%$ (22-24). The combination of doxorubicin with 
ifosfamide is more effective, achieving higher response rates than doxorubicin alone; however, it is associated with severe short-and long-term toxicities, including cardiomyopathy and bone marrow suppression (25-27). Unfortunately, trabectedin, which had been approved as a promising second-line cytostatic agent in the treatment of patients with disseminated soft tissue sarcomas, could not achieve significant higher response rates than doxorubicin in a recently published phase III trial (28).

Due to the rarity and heterogeneity of liposarcomas, further investigations assessing the mechanisms leading to the distinct chemoresistance are still lacking. In recent experimental studies on other solid malignancies, the tumor microenvironment and particularly, tumor-associated fibroblasts (TAFs), were identified as a potential perpetrator of chemoresistance. Two different mechanisms could be observed here. Firstly, TAFs can modulate the chemosensitivity of malignant cells through the secretion of certain cytokines. In an extensive in vitro study, Straussman et al determined the pro-tumorigenic influence of 35 different cytokines on 45 malignant cell lines that had been co-cultured with TAFs (29). Interestingly, $65 \%$ of all treatment groups displayed a diminished chemotherapeutic response that was mediated by TAF-secreted cytokines. Since then, further in vitro studies have revealed a similar cytokine-mediated chemoresistance in breast, lung and pancreas carcinoma cells (30-32). A second mechanism of TAF-induced chemoresistance was detected by Loeffler et al (33). The authors hypothesized that chemotherapeutic drug uptake may be dictated by the interstitial tumor pressure, which in turn is maintained by intratumoral collagen type I. Subsequently, they targeted TAFs as a primary source for collagen type I to decrease the interstitial tumor pressure. By specific in vivo targeting of TAFs via immunotherapy, doxorubicin-intake in breast carcinoma cells was enhanced up to $70 \%$, resulting in marked suppression of tumor growth (33).

Apart from their influence on chemosensitivity, TAFs have also been found to promote the growth and invasive potential of tumor cells by the secretion of cytokines and the remodelling of the extracellular matrix, leading to an increased malignancy in many types of cancer, such as rhabdomyosarcoma, breast, colon and prostate carcinoma (34-39). To date, the crosstalk between host and cancer cells via cytokines with effects on both partners is still unknown in liposarcoma. In the present study, we suggested that there may be a bidirectional crosstalk between liposarcoma cells and TAFs, which may lead to increased proliferation and chemoresistance via soluble mediators. Therefore, we determined the proliferation and doxorubicin sensitivity in a well-established liposarcoma cell line (SW872) following co-culture with primary TAFs obtained from surgically resected liposarcomas.

\section{Materials and methods}

Ethics statement. This study was reviewed and approved by the Ethics Committee of the BG University Hospital Bergmannsheil, Ruhr-University Bochum, Germany with the permit no. 5078-14. All participating patients gave their written informed consent.

TAFs. Tissues for primary cultures of TAFs were collected from one patient with an intermediate-grade myxoid/round cell liposarcoma and a high-grade pleomorphic liposarcoma and one patient with a high-grade pleomorphic liposarcoma that were completely resected for curative intent at our institution. Normal fibroblasts (NFs) were surgically obtained from the dermal layer of both patients. The harvested tissues were minced into small sections, washed with phosphate-buffered saline (PBS) several times and digested with collagenase type I and hyaluronidase (Sigma-Aldrich, St. Louis, MO, USA). Following filtration and centrifugation at $400 \mathrm{x} \mathrm{g}$ for $5 \mathrm{~min}$ at $23^{\circ} \mathrm{C}$, the cells were placed in Dulbecco's modified Eagle's medium (DMEM; Biochrom $\mathrm{GmbH}$, Berlin, Germany) supplemented with $20 \%$ fetal calf serum (FCS; PAN-Biotech GmbH, Aidenbach, Germany), $1 \%$ penicillin $(100 \mathrm{U} / \mathrm{ml})$, streptomycin $(100 \mu \mathrm{g} / \mathrm{ml})$ and $1 \%$ L-glutamine (Sigma-Aldrich). The cells were cultured in a humidified atmosphere at $37^{\circ} \mathrm{C}$ with $5 \% \mathrm{CO}_{2}$ in $25 \mathrm{~cm}^{2}$ flasks. The medium was changed every second day. The experiments were conducted in early passages (3-4 following isolation). $\alpha$-smooth muscle actin ( $\alpha$-SMA) is a well-established marker to detect TAFs in tumor tissue $(34,40,41)$. In our study, the isolated TAFs were examined by immunofluorescence using $\alpha$-SMA antibody (Abcam, Cambridge, UK) at the second passage to confirm the fibroblastic origin of the isolated cells.

Histopathological classification. Both tumors were diagnosed by the Institute of Pathology at the Ruhr University of Bochum. The Institute of Pathology and the Department of Plastic Surgery are part of the Sarcoma Reference Center at the Ruhr University of Bochum. All pathological slides were analyzed or reviewed for consensus diagnosis by experienced soft tissue pathologists.

Liposarcoma cell line and co-culture. The human liposarcoma cells, SW872, were purchased from the American Type Culture Collection (ATCC, Wesel, Germany; cell line CCI-121) and maintained in DMEM medium supplemented with $10 \%$ FCS, $1 \%$ penicillin $(100 \mathrm{U} / \mathrm{ml}), 1 \%$ streptomycin $(100 \mu \mathrm{g} / \mathrm{ml})$ and $1 \%$ L-glutamine. The cells were cultured in a humidified atmosphere at $37^{\circ} \mathrm{C}$ with $5 \% \mathrm{CO}_{2}$ in $25 \mathrm{~cm}^{2}$ flasks. For co-culture, $5 \times 10^{4} \mathrm{SW} 872$ cells were seeded in 12-well co-culture plates (Corning Life Sciences, Acton, MA, USA) in DMEM medium supplemented with $10 \%$ complete chemically defined serum substitute PANEXIN NTA (PAN-Biotech), penicillin $(100 \mathrm{U} / \mathrm{ml}), 1 \%$ streptomycin $(100 \mu \mathrm{g} / \mathrm{ml})$ and $1 \%$ L-glutamine. After $10 \mathrm{~min}$ Transwells with a pore size of $0.4 \mu \mathrm{m}$ and $12 \mathrm{~mm}$ diameter (Corning Life Sciences) were inserted. Subsequently, $4 \times 10^{4}$ TAFs were seeded in each Transwell insert and co-cultured with the SW872 cells for $48 \mathrm{~h}$ before the subsequent experiments were performed.

Cell viability assay. The metabolic activity was measured via 3-(4,5-dimethylthiazol-2-yl)-2,5-diphenyltetrazolium bromide (MTT) assay according to the standard protocol. SW872 cells were grown for $48 \mathrm{~h}$ in co-culture with TAFs or NFs. Subsequently, the SW872 cells were seeded in 96-well microtiter plates (Corning Inc., New York, NY, USA) in a concentration of $3 \times 10^{4}$ cells/well. The following day, the cells were incubated in serum-free medium. Thereafter, the cells were incubated in fresh medium containing MTT solution for a further $4 \mathrm{~h}$. Vital cells integrated the dye as a sign of active metabolism (glycolysis rate). The cells were lysed with dimethyl sulfoxide (DMSO) (Carl Roth GmbH, Karlsruhe, Germany) 
NFs
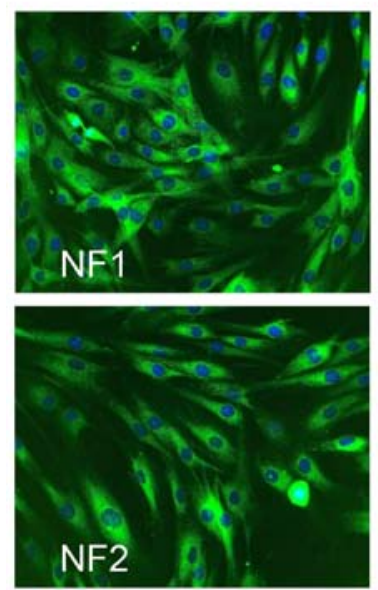

$10 \%$ FCS

Constant cell density

Constant $\alpha$-SMA density
TAFs
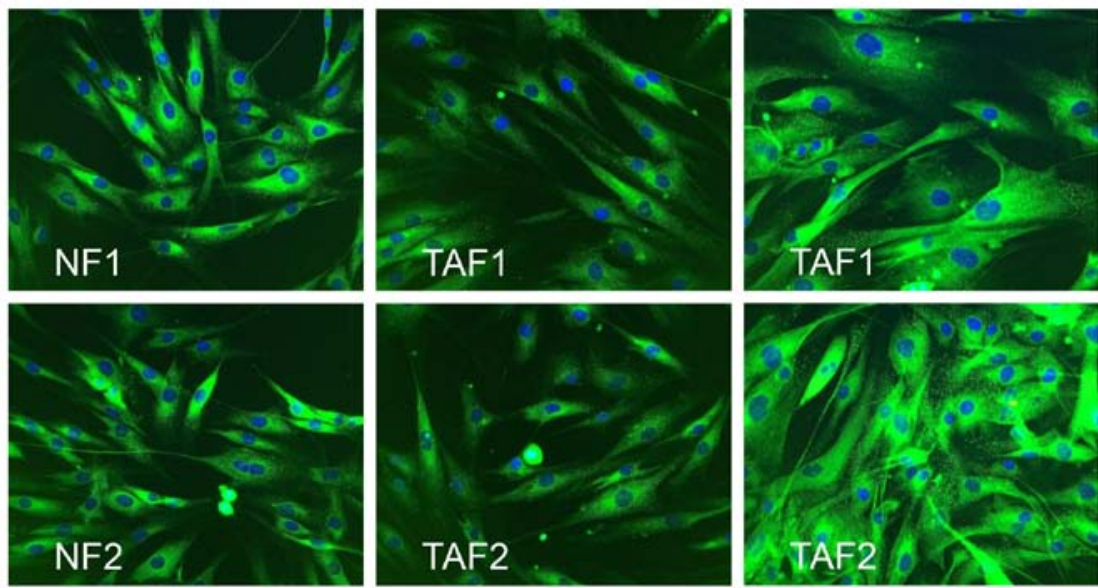

$20 \%$ FCS
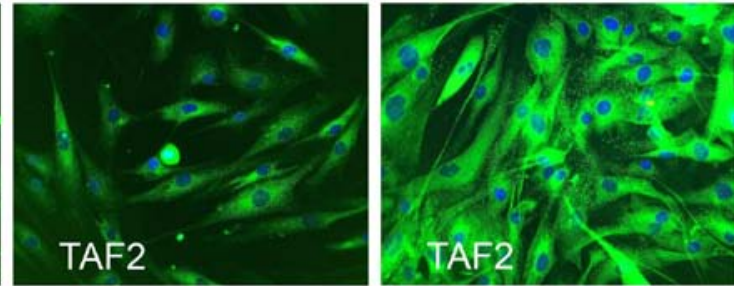

$10 \%$ FCS

$20 \%$ FCS

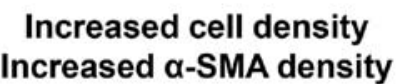

Figure 1. Immunofluorescence detection of $\alpha$-smooth muscle actin ( $\alpha$-SMA) in isolated normal fibroblasts (NFs) and tumor-associated fibroblasts (TAFs). NFs originated from dermal tissue and TAFs from liposarcomas. TAFs expressed $\alpha$-SMA. TAF1 were obtained from intermediate-grade myxoid/round cell liposarcoma and TAF2 from high-grade pleomorphic liposarcoma. Notably, the increase in the FCS concentration led to an enhanced expression of $\alpha$-SMA in TAFs. Original magnification, $\mathrm{x} 200$.

and glycine buffer. The amount of integrated dye represented the level of metabolism and was quantified at $562 \mathrm{~nm}$ using an Elx808 Ultra Microplate Reader (BioTek Instruments GmbH, Bad Friedrichshall, Germany).

Cell proliferation assay. To determine and quantify the effects of TAF co-culture on SW872 cell proliferation, a colorimetric cell proliferation 5-bromo-2'-deoxyuridine (BrdU)-ELISA (Roche Applied Science, Mannheim, Germany) assay was performed according to the manufacturer's instructions. Briefly, the SW872 cells that were grown for $48 \mathrm{~h}$ in co-culture were seeded at $3 \times 10^{4}$ cells/well in 96-well plates. BrdU labeling solution was added followed by incubation for a further $4 \mathrm{~h}$. Proliferating cells integrated BrdU, a pyrimidine analogue, into their DNA. The level of proliferation was quantified by the light emission detected using an Orion microplate luminometer (Berthold Detection Systems GmbH, Pforzheim, Germany).

Real-time cell analysis. For chemosensitivity analysis, the SW872 cells were incubated in co-culture with TAFs or NFs for $48 \mathrm{~h}$. Thereafter, the SW872 cells were seeded in two 8-well plates with an integrated microelectronic sensor array (iCELLigence Real-Time cell analyzer; CEA Biosciences, San Diego, USA). After $24 \mathrm{~h}$, the cultivated human liposarcoma cells were incubated with doxorubicin at a concentration of $0.25 \mu \mathrm{g} / \mathrm{ml}$ that exhibited strong pro-apoptotic effects in previous a in vitro study (42). Cell proliferation and survival were monitored realtime by measuring cell-to-electrode responses of the seeded cells. The cell index (CI) was calculated for each E-plate well using RTCA software 1.2 (Roche Diagnostics, Meylan, France), as previously described (43). CI was calculated for each E-plate well using RTCA Software. The graphs are real-time generated outputs from the iCELLigence system.
Analysis of cell morphology. The morphology of the adherent and suspended cells was examined and documented using a phase contrast Zeiss Axiovert 25 microscope (Carl Zeiss, Jena, Germany).

Statistical analysis. Comparisons between the experimental groups in BrdU- and MTT-assay were performed using one-way measures of variance (one way ANOVAs) over all time points (Tukey test). The results were considered statistically significant for $\mathrm{p}$-values $\leq 0.05$.

\section{Results}

TAFs exhibit a dynamic expression of $\alpha-S M A$. Both, TAFs and NFs expressed $\alpha$-SMA at the second passage, indicating the effective isolation and cultivation of TAFs from tumor tissue (Fig. 1). The morphology of the TAFs obtained from intermediate-grade (TAF1) and high-grade liposarcoma (TAF2) tumor samples did not differ from that of the NFs. They displayed a similar spindle-shaped, elongated and flattened phenotype with few protrusions and small lamellae. The TAFs and NFs exhibited a comparable $\alpha$-SMA expression when cultivated with medium containing $10 \%$ FCS. Notably, the enhancement of the FCS concentration up to $20 \%$ for $24 \mathrm{~h}$ led to an increase in $\alpha$-SMA expression in the TAFs, while the NFs did not respond to this change in the FCS concentration in this short time period (Fig. 1).

Co-culture with TAFs leads to a significantly increased cell viability of SW872 human liposarcoma cells. MTT assay revealed a markedly enhanced metabolic activity of SW872 liposarcoma cells when co-cultured with TAFs (Fig. 2). The SW872 cells that were co-cultured with TAF2 from more malignant high-grade liposarcoma displayed a significantly 


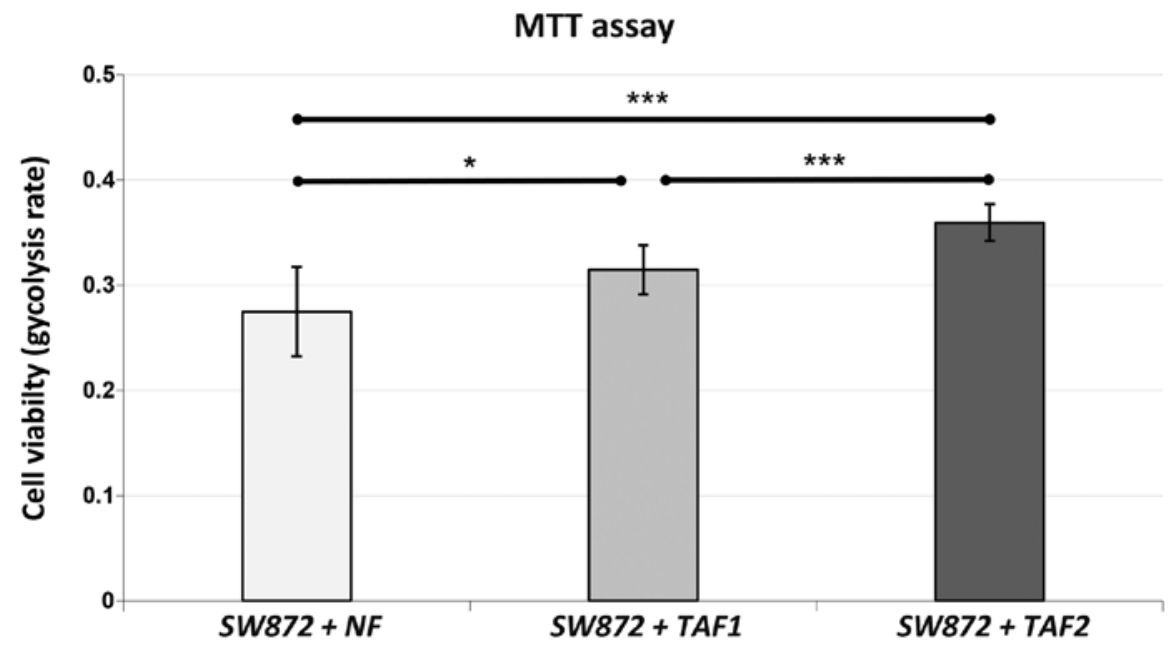

Figure 2. MTT assay. SW872 liposarcoma cells were pre-treated with normal fibroblasts (NFs), tumor-associated fibroblasts (TAFs)1 and TAF2 in transwell cocultures for $48 \mathrm{~h}$. TAF1 were obtained from intermediate-grade myxoid/round cell liposarcoma and TAF2 from high-grade pleomorphic liposarcoma. Subsequently, the viability of the pre-treated SW872 was measured by MTT assay. The assay was performed following $4 \mathrm{~h}$ of incubation. Data are presented as the means \pm SD of 16 measurements. ANOVA was used to demonstrate statistical significance between the different categories with a Tukey's multiple comparison post hoc test. Significantly different at ${ }^{* * * *} \mathrm{p} \leq 0.001,{ }^{*} \mathrm{p}<0.05$. The indicators of significance refer to the differences between the NFs as a control series and the other groups.

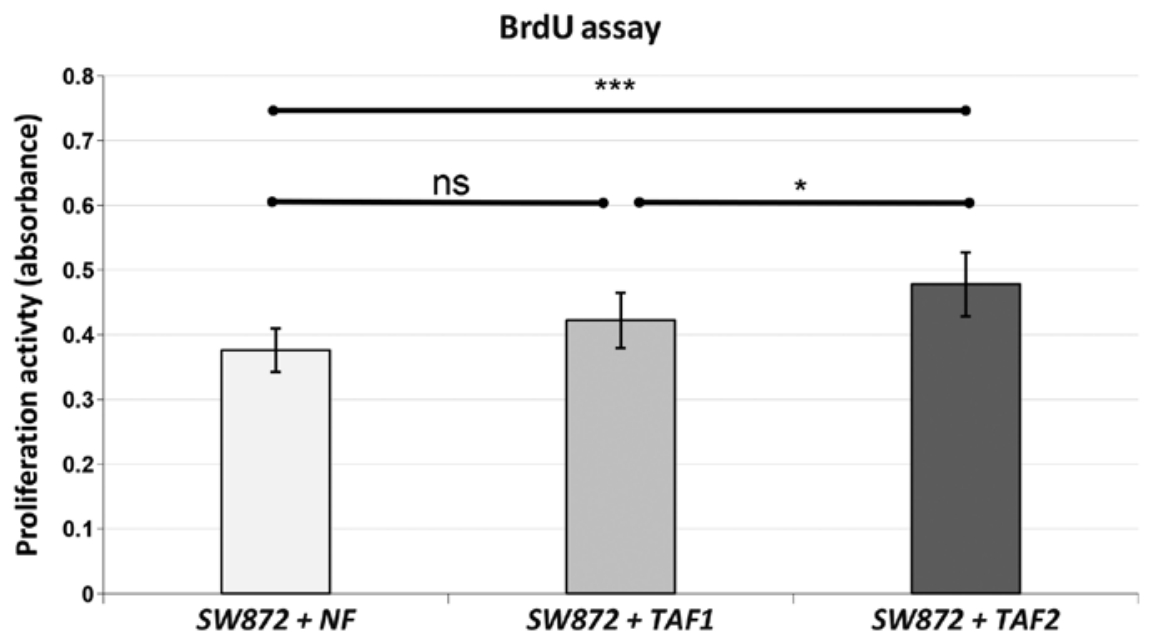

Figure 3. BrdU assay. SW872 liposarcoma cells were pre-treated with normal fibroblasts (NFs), tumor-associated fibroblasts (TAFs) 1 and TAF2 in Transwell co-cultures for $48 \mathrm{~h}$. TAF1 were obtained from intermediate-grade myxoid/round cell liposarcoma and TAF2 from high-grade pleomorphic liposarcoma. The proliferation of the pre-treated SW872 was measured by BrdU assay. The assay was performed following $4 \mathrm{~h}$ of BrdU-incubation. Data are presented as the means \pm SD of 10 measurements. ANOVA was used to demonstrate statistical significance between the different categories with a Tukey's multiple comparison post hoc test. Significantly different at ${ }^{* * *} \mathrm{p} \leq 0.001,{ }^{*} \mathrm{p}<0.05 ; \mathrm{ns}, \mathrm{p}>0.05$. The indicators of significance refer to the difference between NFs as a control series and the other groups.

increased cell viability when compared with the controls cultured with NFs $(\mathrm{p}<0.001)$ or with the cells cultured with TAF1 $(\mathrm{p}=0.001)$ from less malignant intermediate-grade liposarcoma. Nevertheless, co-culture with TAF1 was also associated with a significantly increased cell viability when compared to co-culture with the NFs $(p=0.010)$. Hence, both TAF subgroups were able to enhance the viability of the SW872 cells significantly after $48 \mathrm{~h}$ of co-culture.

The proliferation of SW872 liposarcoma cells is significantly enhanced following co-culture with TAFs from high-grade liposarcoma, but not from intermediate-grade liposarcoma. As indicated by BrdU assay, the proliferative potency of the SW872 cells was significantly increased following co-culture with TAF2 when compared to co-culture with NFs $(\mathrm{p}<0.001)$ or TAF1 ( $\mathrm{p}=0.019$ ) (Fig. 3). Co-culture with TAF1 failed to reach a statistically significant enhancement of proliferation compared to co-culture with NFs $(\mathrm{p}=0.081)$.

Doxorubicin treatment is less effective in SW872 cells co-cultured with TAFs from high-grade liposarcoma. The SW872 cells were pre-treated with different co-cultures of NFs, $T A F 1$ and TAF2. Thereafter, the SW872 cells were seeded in plates with an integrated microelectronic sensor array. After $24 \mathrm{~h}$, doxorubicin was applied to the treatment groups. The viability and proliferation of the SW872 cells were monitored before and during doxorubicin incubation in real-time (Fig. 4). The CI which reflects the number of viable cells initially increased in all treatment groups, but began to decrease after $15 \mathrm{~h}$ of incubation with doxorubicin. Remarkably, the CI of the 

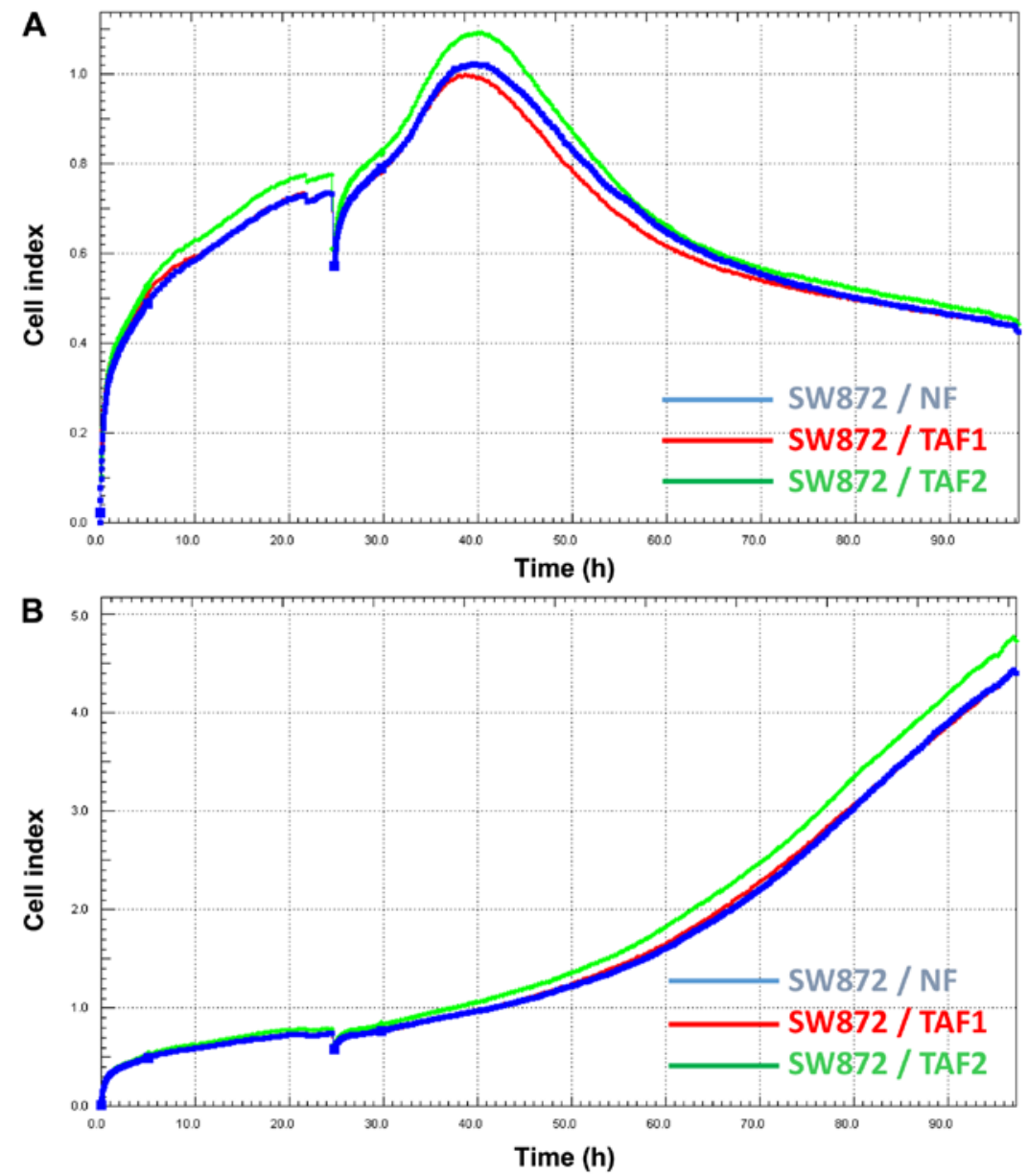

Figure 4. Real-time analysis of SW872 cell viability during (A) doxorubicin treatment and (B) untreated conditions. SW872 liposarcoma cells were pre-treated with normal fibroblasts (NFs), tumor-associated fibroblasts (TAFs) 1 and TAF2 in Transwell co-cultures for $48 \mathrm{~h}$. TAF1 were obtained from intermediategrade myxoid/round cell liposarcoma and TAF2 from high-grade pleomorphic liposarcoma. Thereafter, pre-treated SW872 cells were seeded in 8-well plates with an integrated microelectronic sensor array. The cell index (CI) reflecting the number of viable cells was monitored continuously in real-time. Doxorubicin $(0.25 \mu \mathrm{g} / \mathrm{ml})$ was applied to the wells after $24 \mathrm{~h}$ resulting in a bend of the CI curve in all groups because impedance measurements were transiently disrupted by (A) the addition of doxorubicin solution. CI initially increased, but began to decrease after $15 \mathrm{~h}$ of incubation with doxorubicin in all groups, whereas (B) the untreated cells displayed a steady increase in CI. Notably, CI of cells co-cultured with TAF2 continuously remained at higher levels in the doxorubicin- and the untreated group as well.

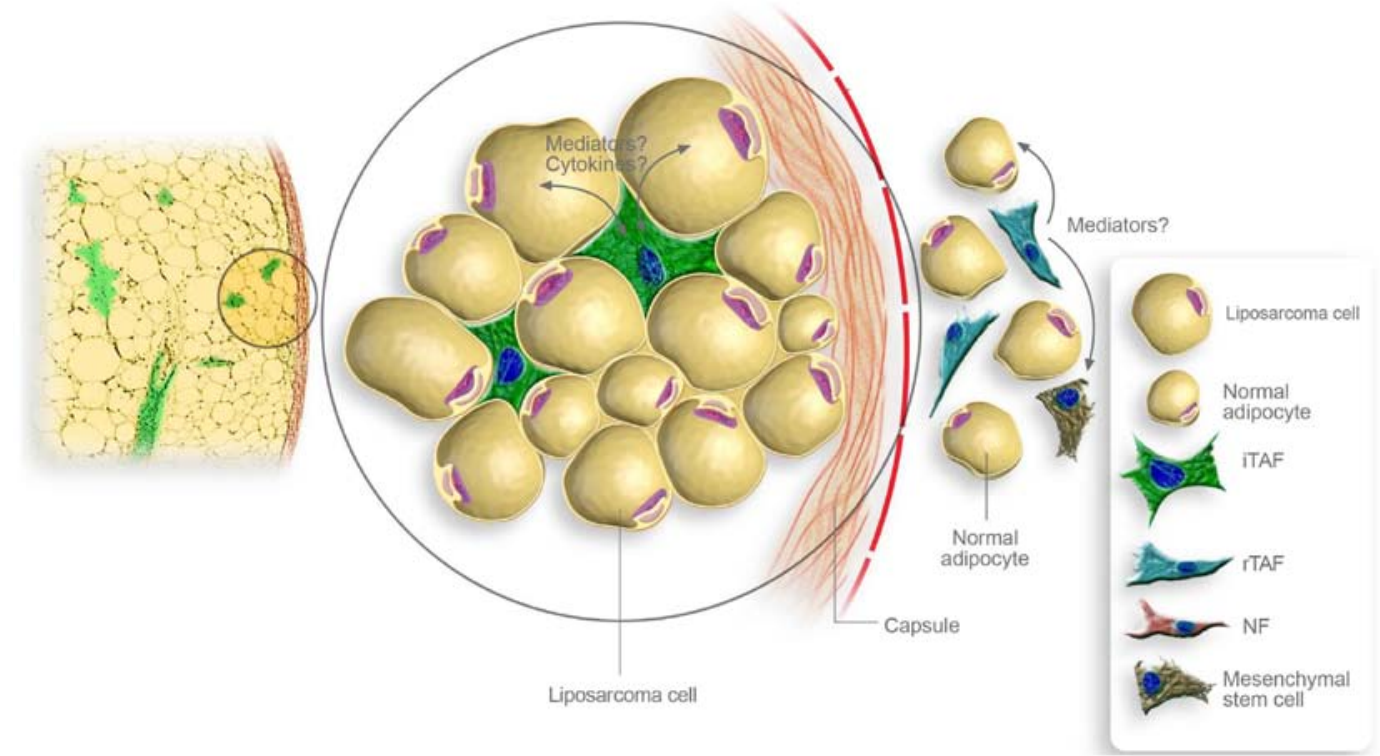

Figure 5. Schematic representation of potential interaction mechanisms provoked by stromal fibroblasts within liposarcomas. Intratumoral tumor-associated fibroblasts (iTAFs) may enhance tumor growth by direct paracrine stimulation of tumor cells through cytokines. Adjacent to the tumor capsule, TAFs at the resection margins (rTAFs) may promote the growth of viable liposarcoma cells located at the tissue borders. They also may alter local mesenchymal stem cells and non-malignant adipocytes facilitating the development of local recurrences. 
SW872 cells which had been co-cultured previously with TAF2 diminished more slightly than that of the cells that had been co-cultured with NFs and TAF1. Finally, the CI of the cells co-cultured with TAF2 continuously remained at higher levels.

\section{Discussion}

Liposarcomas are rare tumors within the heterogeneous group of soft tissue sarcomas and respond poorly to conventional treatments, such as chemotherapy and radiation. Due to the rarity of liposarcomas, the development of novel therapeutics has been difficult, and the lack of novel chemotherapy protocols remains a major obstacle. The mainstay of therapy still implies complete surgical resection with the attainment of microscopically negative margins (R0 resection). Nevertheless, the rates of local recurrence in spite of complete resection are conspicuously high in liposarcomas and cannot be explained by our current knowledge.

There have been several attempts to explain the high rates of local recurrence (14). One potential explanation includes that recurring tumors derive from micrometastases in the tissue around the original location which were left in spite of previous $\mathrm{R} 0$ resection and may be selected out from biologically more aggressive tumor cells which display higher invasive potential and, thus, facilitating local recurrence. Another interesting hypothesis implies that altered but non-malignant cells at the original location, such as TAFs may promote the growth of viable but primarily histological inapparent residual disease in the post-resection tumor bed. In the present study, we demonstrated that TAFs were able to promote the proliferation and viability of liposarcoma cells. Therefore, it seems reasonable that TAFs may ensure the survival of remaining liposarcoma cells in the post-resection tumor bed, leading to local recurrence. In this context, further analyses should assess not only the pro-tumorigenic effects of intratumoral TAFs, but also of TAFs that were adjacent to the tumor and remain in the post-resection tumor bed despite complete tumor resection (Fig. 5).

Interestingly, our experiments indicated that TAFs from more malignant high-grade pleomorphic liposarcoma facilitated the proliferation and viability of liposarcoma cells more strikingly than TAFs from less malignant intermediate-grade myxoid/round cell liposarcoma. Thus, histological tumor grading may not only reflect the aggressiveness of the tumor cells, but may also correlate with the pro-tumorigenic features of the inherent TAFs. Vice versa, TAFs may acquire stronger tumor-promoting properties when growing in more malignant tumor tissues. Remarkably, TAFs from more malignant liposarcoma were able to decrease the chemosensitivity of SW872 liposarcoma cells. Due to our experimental design, the influence of TAFs on the proliferation, viability and chemosensitivity of SW872 liposarcoma cells was mediated through soluble factors that came into contact with the liposarcoma cells during previous co-culture and led to the sustaining alterations. Notably, NFs did not affect the proliferation or drug resistance of liposarcoma cells, indicating a complex bidirectional crosstalk between inherent TAFs and liposracoma cells.

The tumor microenvironment and the involved mediators deserve further investigation in liposarcomas. The apparent contribution to development of drug resistance and tumor progression make TAFs a potential therapeutic target. Present studies are focusing on preclinical TAF-targeting approaches. In contrast to tumor cells, TAFs represent a genetic stable target which may be advantageous for immunotherapeutic approaches $(44,45)$. Further understanding of the involved mediators also enables therapeutic intervention via adjusted antigen body treatment (46). After identification of the involved mediators the corresponding intracellular pathways leading to chemoresistance and tumor progression in liposarcoma cells may be determined and approached.

In conclusion, the present in vitro study demonstrated that TAFs were able to promote the proliferation, viability and drug resistance of liposarcoma cells. To our knowledge, this is the first study that provides evidence for the tumor-progressive influence of TAFs in liposarcoma. The encouraging results of this study suggest experimental support for further in vitro studies with larger cohorts involving all four different liposarcoma subgroups.

\section{References}

1. Hoos A, Lewis JJ and Brennan MF: Soft tissue sarcoma: prognostic factors and multimodal treatment. Chirurg 71: 787-794, 2000 (In German).

2. Mack TM: Sarcomas and other malignancies of soft tissue, retroperitoneum, peritoneum, pleura, heart, mediastinum, and spleen. Cancer 75 (Suppl 1): 211-244, 1995.

3. Dalal KM, Kattan MW, Antonescu CR, Brennan MF and Singer S: Subtype specific prognostic nomogram for patients with primary liposarcoma of the retroperitoneum, extremity, or trunk. Ann Surg 244: 381-391, 2006.

4. Kransdorf MJ: Malignant soft-tissue tumors in a large referral population: distribution of diagnoses by age, sex, and location. AJR Am J Roentgenol 164: 129-134, 1995.

5. Stojadinovic A, Leung DH, Hoos A, Jaques DP, Lewis JJ and Brennan MF: Analysis of the prognostic significance of microscopic margins in 2,084 localized primary adult soft tissue sarcomas. Ann Surg 235: 424-434, 2002.

6. Kaushal A and Citrin D: The role of radiation therapy in the management of sarcomas. Surg Clin North Am 88: 629-646, viii, 2008.

7. Singer S, Demetri GD, Baldini EH and Fletcher CD: Management of soft-tissue sarcomas: an overview and update. Lancet Oncol 1: $75-85,2000$.

8. Collin C, Godbold J, Hajdu S and Brennan M: Localized extremity soft tissue sarcoma: an analysis of factors affecting survival. J Clin Oncol 5: 601-612, 1987.

9. Pisters PW and Pollock RE: Staging and prognostic factors in soft tissue sarcoma. Semin Radiat Oncol 9: 307-314, 1999.

10. Gaynor JJ, Tan CC, Casper ES, Collin CF, Friedrich C, Shiu M, Hajdu SI and Brennan MF: Refinement of clinicopathologic staging for localized soft tissue sarcoma of the extremity: a study of 423 adults. J Clin Oncol 10: 1317-1329, 1992.

11. Pisters PW, Leung DH, Woodruff J, Shi W and Brennan MF: Analysis of prognostic factors in 1,041 patients with localized soft tissue sarcomas of the extremities. J Clin Oncol 14: 1679-1689, 1996.

12. Matsumoto S, Ahmed AR, Kawaguchi N, Manabe J and Matsushita Y: Results of surgery for malignant fibrous histiocytomas of soft tissue. Int J Clin Oncol 8: 104-109, 2003.

13. Lewis JJ, Leung D, Heslin M, Woodruff JM and Brennan MF: Association of local recurrence with subsequent survival in extremity soft tissue sarcoma. J Clin Oncol 15: 646-652, 1997.

14. Daigeler A, Zmarsly I, Hirsch T, Goertz O, Steinau HU, Lehnhardt $\mathrm{M}$ and Harati K: Long-term outcome after local recurrence of soft tissue sarcoma: a retrospective analysis of factors predictive of survival in 135 patients with locally recurrent soft tissue sarcoma. Br J Cancer 110: 1456-1464, 2014.

15. Spillane AJ, Fisher C and Thomas JM: Myxoid liposarcoma - the frequency and the natural history of nonpulmonary soft tissue metastases. Ann Surg Oncol 6: 389-394, 1999.

16. Ghadimi MP, Al-Zaid T, Madewell J, Peng T, Colombo C, Hoffman A, Creighton CJ, Zhang Y, Zhang A, Lazar AJ, et al: Diagnosis, management, and outcome of patients with dedifferentiated liposarcoma systemic metastasis. Ann Surg Oncol 18: 3762-3770, 2011. 
17. Hoffman A, Ghadimi MP, Demicco EG, Creighton CJ, Torres K, Colombo C, Peng T, Lusby K, Ingram D, Hornick JL, et al: Localized and metastatic myxoid/round cell liposarcoma: clinical and molecular observations. Cancer 119: 1868-1877, 2013.

18. Billingsley KG, Lewis JJ, Leung DH, Casper ES, Woodruff JM and Brennan MF: Multifactorial analysis of the survival of patients with distant metastasis arising from primary extremity sarcoma. Cancer 85: 389-395, 1999.

19. Billingsley KG, Burt ME, Jara E, Ginsberg RJ, Woodruff JM, Leung DH and Brennan MF: Pulmonary metastases from soft tissue sarcoma: analysis of patterns of diseases and postmetastasis survival. Ann Surg 229: 602-610; discussion 610-612, 1999.

20. Van Glabbeke M, van Oosterom AT, Oosterhuis JW, Mouridsen H, Crowther D, Somers R, Verweij J, Santoro A, Buesa $J$ and Tursz T: Prognostic factors for the outcome of chemotherapy in advanced soft tissue sarcoma: an analysis of 2,185 patients treated with anthracycline-containing first-line regimens - a European Organization for Research and Treatment of Cancer Soft Tissue and Bone Sarcoma Group Study. J Clin Oncol 17: 150-157, 1999.

21. Italiano A, Toulmonde M, Cioffi A, Penel N, Isambert $N$, Bompas E, Duffaud F, Patrikidou A, Lortal B, Le Cesne A, et al: Advanced well-differentiated/dedifferentiated liposarcomas: role of chemotherapy and survival. Ann Oncol 23: 1601-1607, 2012.

22. Italiano A, Garbay D, Cioffi A, Maki RG and Bui B: Advanced pleomorphic liposarcomas: clinical outcome and impact of chemotherapy. Ann Oncol 23: 2205-2206, 2012.

23. Jones RL, Fisher C, Al-Muderis O and Judson IR: Differential sensitivity of liposarcoma subtypes to chemotherapy. Eur J Cancer 41: 2853-2860, 2005.

24. Patel SR, Burgess MA, Plager C, Papadopoulos NE, Linke KA and Benjamin RS: Myxoid liposarcoma. Experience with chemotherapy. Cancer 74: 1265-1269, 1994.

25. Brodowicz T, Schwameis E, Widder J, Amann G, Wiltschke C, Dominkus M, Windhager R, Ritschl P, Pötter R, Kotz R and Zielinski CC: Intensified adjuvant IFADIC chemotherapy for adult soft tissue sarcoma: a prospective randomized feasibility trial. Sarcoma 4: 151-160, 2000.

26. Frustaci S, Gherlinzoni F, De Paoli A, Bonetti M, Azzarelli A, Comandone A, Olmi P, Buonadonna A, Pignatti G, Barbieri E, et al: Adjuvant chemotherapy for adult soft tissue sarcomas of the extremities and girdles: results of the Italian randomized cooperative trial. J Clin Oncol 19: 1238-1247, 2001.

27. Judson I, Verweij J, Gelderblom H, Hartmann JT, Schöffski P, Blay JY, Kerst JM, Sufliarsky J, Whelan J, Hohenberger P, et al; European Organisation and Treatment of Cancer Soft Tissue and Bone Sarcoma Group: Doxorubicin alone versus intensified doxorubicin plus ifosfamide for first-line treatment of advanced or metastatic soft-tissue sarcoma: a randomised controlled phase 3 trial. Lancet Oncol 15: 415-423, 2014.

28. Blay JY, Casali P, Nieto A, Tanović A and Le Cesne A: Efficacy and safety of trabectedin as an early treatment for advanced or metastatic liposarcoma and leiomyosarcoma. Future Oncol 10: 59-68, 2014

29. Straussman R, Morikawa T, Shee K, Barzily-Rokni M, Qian ZR, Du J, Davis A, Mongare MM, Gould J, Frederick DT, et al: Tumour micro-environment elicits innate resistance to RAF inhibitors through HGF secretion. Nature 487: 500-504, 2012.

30. Rong G, Kang H, Wang Y, Hai T and Sun H: Candidate markers that associate with chemotherapy resistance in breast cancer through the study on Taxotere-induced damage to tumor microenvironment and gene expression profiling of carcinoma-associated fibroblasts (CAFs). PLoS One 8: e70960, 2013.
31. Wang W, Li Q, Yamada T, Matsumoto K, Matsumoto I, Oda M, Watanabe G, Kayano Y, Nishioka Y, Sone S and Yano S: Crosstalk to stromal fibroblasts induces resistance of lung cancer to epidermal growth factor receptor tyrosine kinase inhibitors. Clin Cancer Res 15: 6630-6638, 2009.

32. Hwang RF, Moore T, Arumugam T, Ramachandran V, Amos KD, Rivera A, Ji B, Evans DB and Logsdon CD: Cancer-associated stromal fibroblasts promote pancreatic tumor progression. Cancer Res 68: 918-926, 2008

33. Loeffler M, Krüger JA, Niethammer AG and Reisfeld RA: Targeting tumor-associated fibroblasts improves cancer chemotherapy by increasing intratumoral drug uptake. J Clin Invest 116: 1955-1962, 2006

34. Orimo A, GuptaPB,Sgroi DC,Arenzana-Seisdedos F,Delaunay T, Naeem R, Carey VJ, Richardson AL and Weinberg RA: Stromal fibroblasts present in invasive human breast carcinomas promote tumor growth and angiogenesis through elevated SDF-1/CXCL12 secretion. Cell 121: 335-348, 2005.

35. Lee YS, Choi I, Ning Y, Kim NY, Khatchadourian V, Yang D, Chung HK, Choi D, LaBonte MJ, Ladner RD, et al: Interleukin-8 and its receptor CXCR2 in the tumour microenvironment promote colon cancer growth, progression and metastasis. Br J Cancer 106: 1833-1841, 2012.

36. Olumi AF, Grossfeld GD, Hayward SW, Carroll PR, Tlsty TD and Cunha GR: Carcinoma-associated fibroblasts direct tumor progression of initiated human prostatic epithelium. Cancer Res 59: 5002-5011, 1999.

37. Kalluri R and Zeisberg M: Fibroblasts in cancer. Nat Rev Cancer 6: 392-401, 2006.

38. Tarnowski M, Grymula K, Liu R, Tarnowska J, Drukala J, Ratajczak J, Mitchell RA, Ratajczak MZ and Kucia M: Macrophage migration inhibitory factor is secreted by rhabdomyosarcoma cells, modulates tumor metastasis by binding to CXCR4 and CXCR7 receptors and inhibits recruitment of cancerassociated fibroblasts. Mol Cancer Res 8: 1328-1343, 2010.

39. Mishra P, Banerjee D and Ben-Baruch A: Chemokines at the crossroads of tumor-fibroblast interactions that promote malignancy. J Leukoc Biol 89: 31-39, 2011.

40. Augsten M: Cancer-associated fibroblasts as another polarized cell type of the tumor microenvironment. Front Oncol 4: 62, 2014.

41. Sugimoto H, Mundel TM, Kieran MW and Kalluri R: Identification of fibroblast heterogeneity in the tumor microenvironment. Cancer Biol Ther 5: 1640-1646, 2006.

42. Harati K, Chromik AM, Bulut D, Goertz O, Hahn S, Hirsch T, Klein-Hitpass L, Lehnhardt M, Uhl W and Daigeler A: TRAIL and taurolidine enhance the anticancer activity of doxorubicin, trabectedin and mafosfamide in HT1080 human fibrosarcoma cells. Anticancer Res 32: 2967-2984, 2012.

43. Koval OA, Sakaeva GR, Fomin AS, Nushtaeva AA, Semenov DV, Kuligina EV, Gulyaeva LF, Gerasimov AV and Richter VA: Sensitivity of endometrial cancer cells from primary human tumor samples to new potential anticancer peptide lactaptin. J Cancer Res Ther 11: 345-351, 2015.

44. Kakarla S, Song XT and Gottschalk S: Cancer-associated fibroblasts as targets for immunotherapy. Immunotherapy 4: 1129-1138, 2012

45. Underwood TJ, Hayden AL, Derouet M, Garcia E, Noble F, White MJ, Thirdborough S, Mead A, Clemons N, Mellone M, et al: Cancer-associated fibroblasts predict poor outcome and promote periostin-dependent invasion in oesophageal adenocarcinoma. J Pathol 235: 466-477, 2015.

46. Togo S, Polanska UM, Horimoto Y and Orimo A: Carcinomaassociated fibroblasts are a promising therapeutic target. Cancers (Basel) 5: 149-169, 2013 